\title{
Signal changes on MRI and increases in reactive microgliosis, astrogliosis, and iron in the putamen of two patients with multiple system atrophy
}

\author{
J Schwarz, S Weis, E Kraft, K Tatsch, O Bandmann, P Mehraein, T Vogl, W H Oertel
}

\begin{abstract}
Department of Neurology, Klinikum Grosshadern, LudwigMaximilians-

University, Munich, Germany and BMFT Research Program Munich "Parkinson's disease and other basal ganglia disorders"

J Schwarz

E Kraft

O Bandmann most likely correlated with the area of hyperintense signal changes on MRI. This area also contained the highest amount of ferric iron, which was increased in the putamen of patient 1 but not patient 2 .

It is unlikely that the hypointense signal changes in the putamen are due to an increase of iron alone. Reactive microglial and astroglial cells may play a part in the pathogenesis of MSA.
\end{abstract}

W H Oertel

Institute of

Neuropathology,

Ludwig-Maximilians-

University, Munich,

Germany

$\mathrm{S}$ Weis

Department of

Radiology, Med Klinik Innenstadt, Ludwig-

Maximilians-

University, Munich,

Germany

E Kraft

T Vogl

Department of Nuclear Medicine, Klinikum

Grosshadern, LudwigMaximilians-

University, Munich, Germany

K Tatsch

Correspondence to: Dr Johannes Schwarz, Department of Neurology, Klinikum Grosshadern, Ludwig-Maximilians-

University Munich,

Marchionini Strasse 15,

81377 München, Germany.

Received 3 January 1995

and in revised form

August 1995

Accepted 1 September 1995

\begin{abstract}
A correlation of clinical, MRI, and neuropathological data is reported in two patients with multiple system atrophy (MSA).

On MRI, patient 1 showed striatal atrophy, reduction of $T 2$ relaxation times within most of the putamen, and a band of hyperintense signal changes in the lateral putamen. In patient 2 , MRI disclosed only shortening of the $\mathrm{T} 2$ signal in the putamen.
\end{abstract}

Immunohistochemistry showed pronounced reactive microgliosis and astrogliosis in the affected brain regions. In patient 1 , the area with the most pronounced microgliosis and astrogliosis

(F Neurol Neurosurg Psychiatry 1996;60:98-101)

Keywords: multiple system atrophy; magnetic resonance imaging, astrogliosis; microgliosis; iron deposits

T2 weighted MRI may help in the differential diagnosis of Parkinson's disease and multiple system atrophy of the striatonigral degeneration type. The reduction of the putaminal T2 signal in patients with multiple system atrophy (MSA) or related disorders has mainly been attributed to the deposition of ferric iron. ${ }^{13}$ Neuropathological changes leading to hyperintense signal changes in the lateral part of the putamen may be due to gliosis.

We correlated hypointense and hyperintense signal changes on T2 weighted MRI with loss of neurons, reactive microgliosis, and astrogliosis, and iron content in the putamen in two patients with definite MSA.

\section{Methods}

MAGNETIC RESONANCE IMAGING

Brain MRI was performed with a Siemens
Magnetom scanner operated at 1.5 Tesla (Erlangen, Germany). Images were recorded with a proton density and heavily $\mathrm{T} 2$ weighted spin echo sequence (TR/TE $=2900 / 20-90$ $\mathrm{ms}$ ) in axial and coronal orientation without gap (individual slice thickness $=3 \mathrm{~mm}$ ).

${ }^{123}$ I-iodobenzamide (IBZM) SPECT was performed as reported elsewhere. ${ }^{*}$

\section{NEUROPATHOLOGY}

After fixation in a $4 \%$ formalin solution, various specimens were embedded in paraffin and the following stains were used: haematoxylin and eosin, cresyl violet (Nissl stain), luxol fast blue, Prussian blue, and modified silver impregnation. ${ }^{5}$ Immunohistochemical analyses were performed with the following antibodies: anti-ubiquitin (1:300, polyclonal, DAKO), anti-tau-2 (1:2000, monoclonal, Boehringer), anti- $\beta$-amyloid $\quad(1: 100, \quad$ monoclonal, Boehringer), anti-GFAP (glial fibrillary acid protein) (1:100, monoclonal, DAKO), antiCD68 (1:100, monoclonal, DAKO), antiferritin $(1: 2000$, polyclonal, DAKO), and anti-Cr3/43 (1:100, monoclonal, DAKO).

\section{Case reports}

PATIENT 1

A 41 year old woman presented to our clinic in 1992 with a three year history of rapidly progressive parkinsonism. She also complained about blurred speech, an impaired balance of stance and gait, numerous syncopies after standing up, and urinary incontinence. Neurological examination disclosed symmetric akinetic rigid parkinsonism and a cerebellar syndrome. Plantar responses were extensor bilaterally. One minute after standing up there was a drop in blood pressure (160/90 to $90 / 60$ $\mathrm{mm} \mathrm{Hg}$ ) without a change in heart rate. She had received levodopa up to $600 \mathrm{mg}$ per day without any benefit. She was clinically diagnosed as probable MSA of striatonigral degeneration type. Four weeks later she suddenly died of cardiac arrest at home.

Brain MRI performed three weeks before death showed mild cortical and cerebellar atrophy. There were pronounced hypointense lesions in the putamen on T2 weighted sequences. In the posterolateral part of the putamen, a band of hyperintense signal changes was prominent on $\mathrm{T} 2$ and proton density spin echo sequences (fig 1). Specific 
Figure 1 Transverse reconstruction of $T 2$ weighted MRI sequences at the level of the

posterolateral putamen in patient 1 (left) and patient 2 (right). Black arrows indicate hypointense signal changes in the putamen

(patient 1 and 2), and white arrows indicate

the band of hyperintensity at the border of the lateral putamen and the external capsule, which is more pronounced on the left side (patient 1).
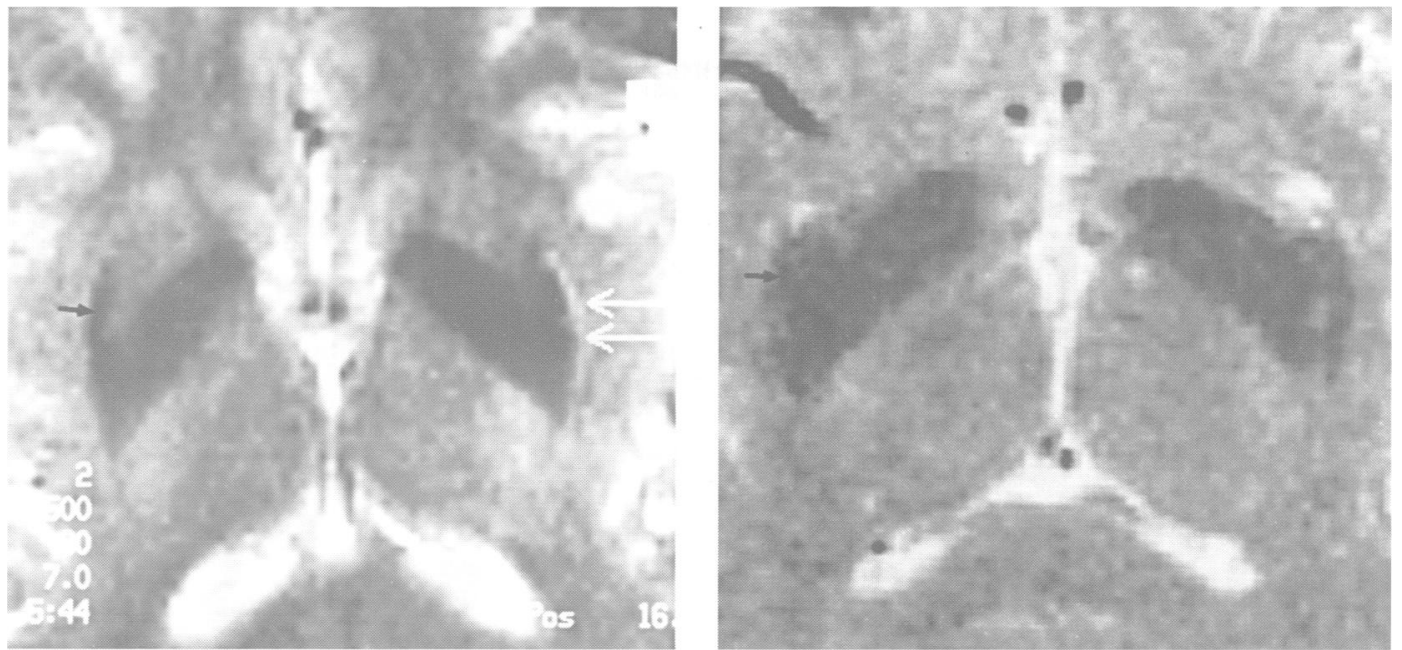

Figure 2 Light

microscopic investigations of the lateral putamen in patient 1 showing: $(A)$

pronounced increase of

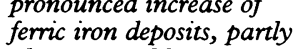
phagocytosed by

macrophages and

astrocytes (Prussian blue,

$12000 \times)$; (B) large

increase of activated

microglialmacrophages

(anti-CR 3/43, $300 \times$ ).

A
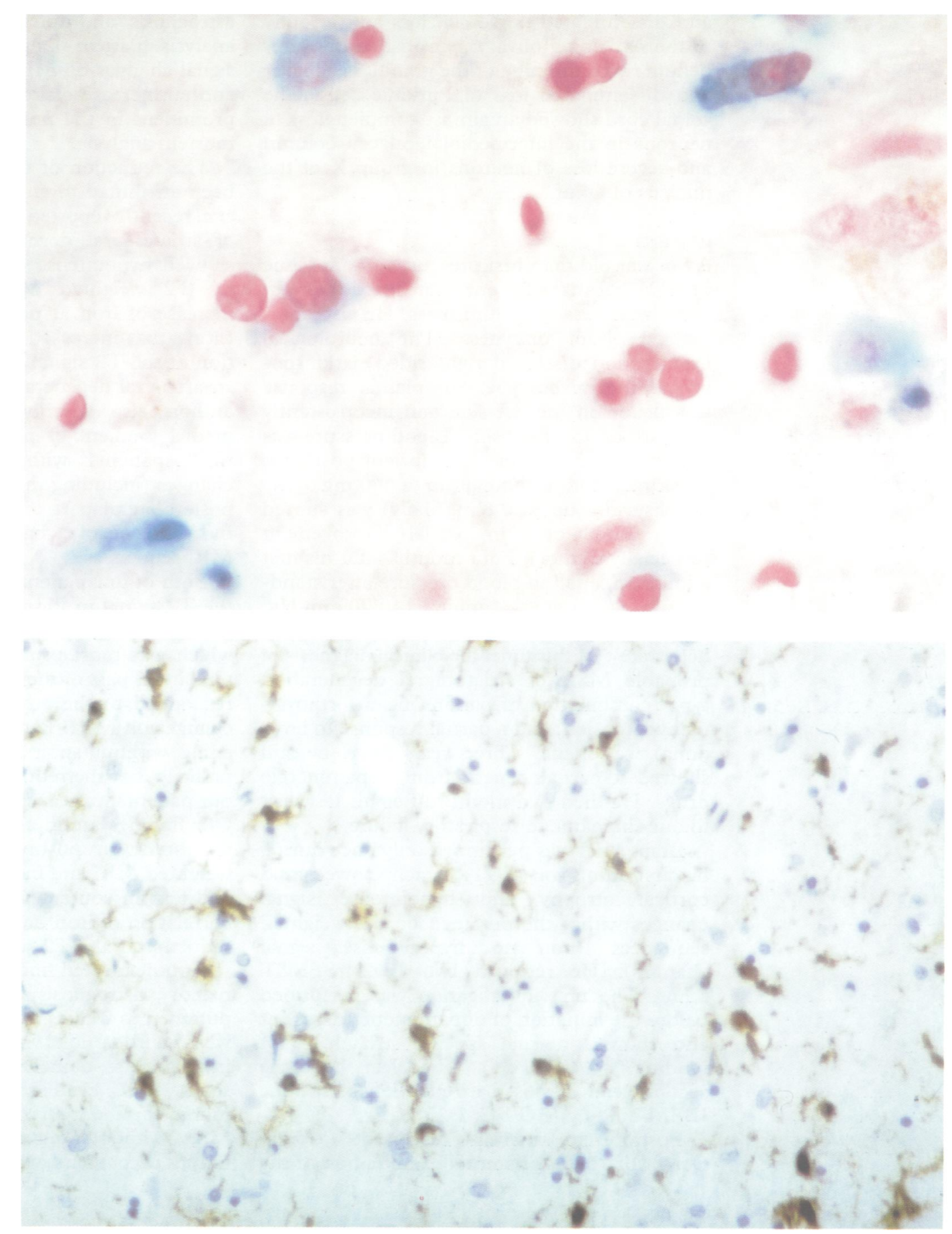
striatal IBZM binding was much reduced $(\mathrm{BG} / \mathrm{FC}: 1 \cdot 31$, normal range $>1 \cdot 45)$.

At postmortem analysis the brain weighed $1220 \mathrm{~g}$. Light microscopic examination of the putamen showed severe nerve cell loss, most prominent in the posterolateral aspects. We also found an increase in extracellular and parenchymal ferric iron deposits, again, mostly in the posterolateral part (fig $2 \mathrm{~A}$ ). In the posterolateral region, a strong reactive astrogliosis and reactive microgliosis (antibodies against $\mathrm{CD} 68$, ferritin, and $\mathrm{Cr} 3 / 43$; fig $2 \mathrm{~B}$ ) were present. The substantia nigra showed mild to moderate loss of pigmented neurons without Lewy bodies. Here we also found parenchymal iron deposits in moderate amount as well as a moderate increase of reactive astroglial and microglial cells. Glial cytoplasmic inclusions were seen in the pons and internal capsule by the modified silver impregnation technique and antiubiquitin immunohistochemistry. The inferior olivary nucleus showed a moderate and disseminated nerve cell loss and atrophy of the remaining olivary neurons. In the cerebellum was a thinning of the granular cell layer and disseminated loss of Purkinje cells. The spinal cord showed an almost complete loss of neurons in the intermediolateral cell column and severe loss of neurons in group $\mathrm{X}$ of the nucleus of Onuf.

PATIENT 2

A 56 year old man first presented to our clinic in 1991 because of a one year history of right sided weakness and clumsiness. He also complained about dizzyness. The neurological examination disclosed right sided parkinsonism of the mixed type. His plantar response was flexor on the left side and inconsistently extensor on the right side. Blood pressure was normal in both supine and upright positions. Treatment with levodopa (up to $600 \mathrm{mg}$ daily) and lisuride (up to $0.8 \mathrm{mg}$ daily) was started but resulted only in partial improvement (especially tremor). Four months after his first admission, a fall in blood pressure after standing up was noted (supine: $140 / 90 \mathrm{~mm} \mathrm{Hg}$; standing up: $90 / 60 \mathrm{~mm} \mathrm{Hg}$; without change of heart rate). At this time, the clinical diagnosis of probable MSA of striatonigral degeneration type (combination of parkinsonism, orthostatic hypotension, and a partial response to levodopa) was made. Three years later, he still showed a partial benefit from dopaminergic drugs. He died two months after his last visit to our clinic due to respiratory failure.

Brain MRI was performed only once during his first admission (fig 1), which showed mild cortical atrophy and hypointense signal changes within the putamen on $\mathrm{T} 2$ weighted sequences (but no hyperintense signal changes). He received two IBZM-SPECT scans. The first investigation was performed before the initiation of dopamimetic treatment and showed normal specific striatal IBZM binding (BG/FC: 1.56). Three years later, the second scan disclosed a reduction of specific IBZM binding (BG/FC: $1 \cdot 25)$.

Postmortem analysis disclosed a brain weight of $1560 \mathrm{~g}$. External examination of the brain showed moderate brain oedema with signs of tonsillar and uncal herniation. The putamen showed moderate nerve cell loss in the posterolateral part with a large increase of reactive astrogliosis and microgliosis. There was no increase of iron deposits. In the substantia nigra, we found loss of pigmented neurons, but no Lewy bodies. In the pons and internal capsule, many argyrophilic and ubiquitin positive glial inclusion bodies were detected. The cerebellum showed a disseminated loss of Purkinje cells. The spinal cord, including group $\mathrm{X}$ of the nucleus of Onuf, showed no changes.

\section{Discussion}

We report a clinicopathological correlation of two patients with MSA of striatonigral degeneration type. In the putamen, both patients showed a reduction of the T2 signal on MRI in vivo, and a pronounced increase of reactive astrogliosis and microgliosis at postmortem analysis. Patient 1 also showed hyperintense signal changes on MRI in the lateral putamen and an increase of iron in the putamen, most prominent in the posterolateral part at postmortem analysis.

The reduction of the T2 signal has mostly been attributed to an increase in iron. ${ }^{23}$ Lang et al recently reported three patients with MSA of striatonigral degeneration type who also showed hypointense changes in the putamen on T2 weighted MRI sequences and an increase of iron at postmortem analysis indicating that increased iron results in a reduction of the T2 signal in patients with MSA of striatonigral degeneration type. ${ }^{2}$ However, the authors also described a striking lateral to medial gradient of putaminal changes in all three patients with the most prominent changes (including the increase in iron) in the posterolateral part of the putamen, and also the presence of a band of hyperintensity on MRI sequences. ${ }^{2}$

Both of our patients showed a reduction of the T2 signal in the putamen. However, only patient 1 also showed an increase in iron, which was most pronounced within the posterolateral part of the putamen. This area corresponded to the area of hyperintense signal changes on MRI. Patient 2 showed T2 shortening without an increase in iron. It seems unlikely that the reduction of the T2 signal in our patients was caused by an increase in iron. Our findings are in agreement with a correlative study in postmortem tissue using T2 weighted MRI and biochemical assays for iron and ferritin content that also failed to show a correlation of iron or ferritin content with the reduction of the T2 signal. ${ }^{6}$

An unexpected finding was the pronounced reactive astrogliosis and microgliosis in the putamen as well as in other areas of the brain, with neuronal loss, in both patients. Changes in glial cells-namely, glial cytoplasmic inclusions-have been recognised as pathognomonic for the neuropathological diagnosis of MSA,${ }^{7}$ although these inclusions were recently found in patients with other diseases. ${ }^{8}$ The 
amount of reactive microgliosis in our patients indicates that glial changes are likely to play a part in the pathogenesis of MSA and may also contribute to the signal changes seen on MRI.

Our results confirm earlier reports of patients with (postmortem) definite MSA, who showed hypointense (and hyperintense) signal changes in the putamen on $\mathrm{T} 2$ weighted MRI in vivo. ${ }^{12}$ Gliosis and iron deposition may both contribute to signal changes seen on MRI. These data also show that pronounced reactive microgliosis and astrogliosis are prominent features of neurodegeneration occurring in patients with MSA of striatonigral degeneration type.

This work was supported by a grant from the German ministry of research and technology (BMFT 01KL9001).
1 O'Brien C, Sung JH, McGeachie RE, Lee MC. Striatonigral degeneration: clinical MRI and pathologic Striatonigral degeneration: clinical M
correlation. Neurology 1990;40:710-1.

2 Lang AE, Curran T, Provias J, Bergeron C. Striatonigral degeneration: iron deposition in putamen correlates with the slit-like void signal of magnetic resonance imaging. Can F Neurol Sci 1994;21:311-8.

3 Olanow CW. Magnetic resonance imaging in parkinsonism. Neurol Clin 1992;10:405-10.

4 Schwarz J, Tatsch K, Vogl T, et al. Marked reduction of striatal dopamine D2 receptors as detected by ${ }^{123}$ I-IBZMSPECT in a Wilson's disease patient with generalized dystonia. Mov Disord 1992;7:58-61.

5 Abe $\mathrm{H}$, Mehrain $\mathrm{P}$, Weis S. A modified NOR-silver impregnation technique for amyloid plaques and neurofibrillary tangles: comparative assessment. Neuropathol Appl Neurobiol 1994;20:478-86.

6 Chen JC, Hardy PA, Kucharczyk W, et al. MR of human brain tissue: a correlative study between T2 and assays of iron and ferritin in Parkinson and Huntington disease. AFNR Am f Neuroradiol 1993;14:275-81.

7 Lantos PL, Papp MI. Cellular pathology of multiple system atrophy: a review. $\mathcal{F}$ Neurol Neurosurg Psychiatry 1994;57: 129-33.

8 Daniel SE, Geddes JF, Revesz T. Glial cytoplasmic inclusions are not exclusive to multiple system atrophy. $\mathcal{F}$ Neurol Neurosurg Psychiatry 1995;58:262. 